\title{
Accelerating 3D Microstructure Acquisition via Fully Automated Serial Sectioning
}

L.T. Nguyen ${ }^{1}$ and D.J. Rowenhorst ${ }^{2}$

${ }^{1}$ National Research Council and U.S. Naval Research Laboratory, Code 6350, Washington, DC, USA

${ }^{2}$ U.S. Naval Research Laboratory, Code 6350, Washington, DC, USA

The three-dimensional (3D) representation of a material contains the essential microstructural, crystallo-graphic, and chemical information that describe its behavior. In many materials systems, the microstruc-ture is polycrystalline with one or more phases and features that may not be captured through only two-dimensional studies. 3D characterization techniques have been rapidly developing across a range of length scales.

While some techniques such as focused ion beam, plasma, and femtosecond laser can obtain data at high resolution (submicron), the volumes of these techniques range from $0.01 \mathrm{~mm}^{3}$ to less than $1 \mathrm{~mm}^{3}$ and cannot reach the length scales needed for statistical significance for many relevant materials problems [1]. Datasets that use techniques such as x-ray tomography are on the multi-millimeter scale, but have poor spatial resolution or a poor contrast mechanism for metallic samples [1]. However, mechanical serial sectioning can examine fields of view over $1 \mathrm{~mm}^{2}$ at high spatial resolutions. With large volumes in excess of $1 \mathrm{~mm}^{3}$, statistically significant microstructural information can be used to explain materials phenomena.

A previous study of 316L stainless steel investigated how processing parameters affect grain boundary networks, which was then serial sectioned [2]. Each section contained backscattered electron images (BEI) and electron backscatter diffraction (EBSD) data with an area of $1 \mathrm{~mm}^{2}$, a spatial resolution of $0.5 \mu \mathrm{m}$, and a section-to-section thickness of $1 \mu \mathrm{m}$. 50 sections were collected for a final volume of $850 \mu \mathrm{m} \times 850 \mu \mathrm{m} \times 50 \mu \mathrm{m}$. This study demonstrated that it is possible to obtain high resolution, large scale $3 \mathrm{D}$ datasets.

Due to the amount of time and human labor required to collect this dataset, the Robotic Serial Sectioning System (RS3D) was developed. Shown in Figure 1(a), RS3D is a fully automated mechanical serial sectioning system similar to the one at the Air Force Research Laboratory [3]. This study shows the results of a 3D investigation of 316L stainless steel using the RS3D, as shown in Figure 1(a). It took one month to collect 50 sections; it previously took one year to manually collect the same volume of data in the previous investigation, which is shown in Figure 1(b).

This study also presents the framework designed to operate the RS3D, which consists of several subsystems that make up one main system. The system uses a human readable text file that contains a checklist of steps, where each step dictates the operation of a subclass. The development included creating a suite of versatile tools held in a python class that have adapted the electron microscope API for the functionality and safety features needed for the full operation of the microscope.

The main goal is to identify the challenges involved with implementing an automated system. This sys-tem has been adapted so that when an error occurs, it stops its current task and informs the user. Some challenges include adequate sample preparation, repeatable sample positioning with a mechanical stage, reliable autofocus and auto brightness/contrast, and intermittent software crashes. 
All the required tools are available to run a fully automated mechanical serial sectioning experiment with minimal user intervention. When fully operational, RS3D can collect six sections in one day, a poten-tial speed up of about 20 times over the manual process. As technology and instrumentation continue to improve, the system is versatile enough to adapt these advancements. Through the design and implemen-tation of RS3D, a fully automated serial sectioning system with submicron resolution and large volume acquisition capabilities is possible [4].

\section{References:}

[1] M.D. Uchic, M.A. Groeber, and A.D. Rollett. JOM, 63 (2011), p. 25.

[2] A.J. Levinson, D.J. Rowenhorst, and A.C. Lewis. JOM, 66, (2014), p. 774.

[3] M.D. Uchic, Report AFRL-RX-WP-TP-2012-0254 (2012).

[4] This work was funded by the National Academies of Science, Engineering, and Medicine and the Naval Research Laboratory under the auspices of the Office of Naval Research and from the Structural Metallics program of ONR.

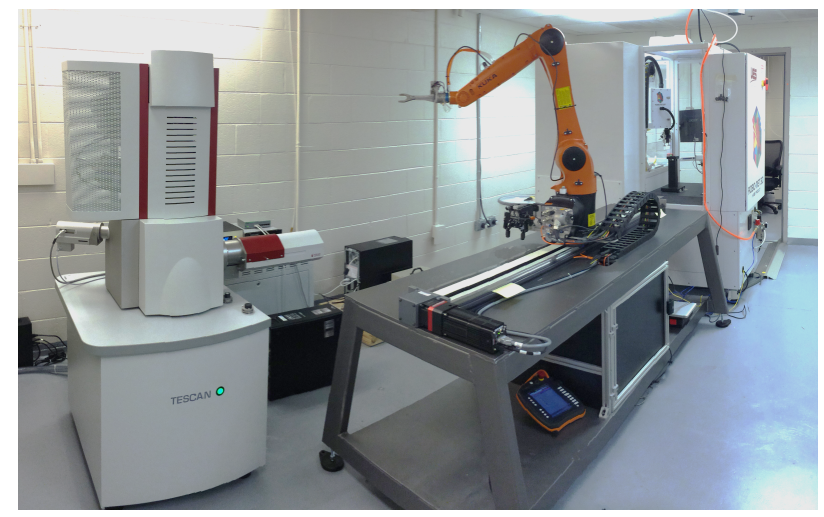

(a)

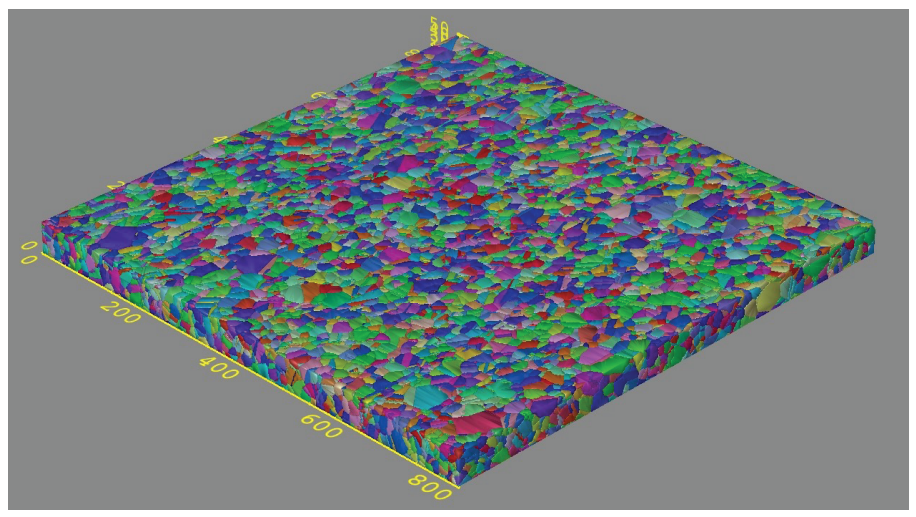

(b)

Figure 1. (a) The setup of the Robotic Serial Sectioning System. From left to right: Tescan MIRA3 scanning electron microscope, Kuka six-axis robot arm, and RoboMet.3D polishing system. (b) A 3D crystallographic representation of 316L stainless steel from a previous investigation. 